64 months \pm 14.31 months. Table 1 Comparison of recurrence rates depending on the characteristics of the tumor process and the type of treatment

Conclusions Our study demonstrates that the frequency of their occurrence is approximately the same, regardless of the method of treatment used. However, with relapses, life expectancy is significantly reduced.

\section{EPV110/\#289 ENDOMETRIAL ASPIRATION FOR ENDOMETRIAL CANCER DIAGNOSIS}

L Barbosa*, B Napoleao, M Ribeiro, M Pagani, V Silveira, R Grande, F Campos. Universidade do Vale do Sapucai, Oncoginecologia, Pouso Alegre, Brazil

10.1136/ijgc-2021-IGCS. 180

Objectives The diagnosis of endometrial cancer, the third most common among gynecological tumors in Brazil, must be made by anatomopathological examination of the biopsy of the endometrial cavity using hysteroscopy or semiotic curettage. Recently, the endometrial aspirate technique has been used in order to speed up the diagnosis as it is an easy, low cost, outpatient method, dispensing with more complex tests, such as hysteroscopy. The aim of the study was to compare the results of this technique with those of semiotic uterine curettage in women with suspected endometrial hyperplasia/carcinoma.

Methods Analytical and retrospective study by analyzing the medical records of 52 women between 41 and 83 years old at the outpatient clinic of Hospital das Clínicas Samuel Libânio, Brazil. Material collected by means of endometrial aspirate and uterine curettage from patients with endometrial thickening on ultrasound, with or without bleeding, uterine bleeding after menopause or abnormal uterine bleeding.

Results 52 patients evaluated with endometrial aspirate, 12 were diagnosed with endometrial adenocarcinoma and three with hyperplasia with endometrial atypia. The endometrial aspirate was positive in 8 of the adenocarcinomas, suspected in two and negative in two other cases. In atypical hyperplasia, aspirate was positive in one case and negative in two.

Conclusions The use of endometrial aspirate for diagnosis was $66.6 \%$ positive in this study, a satisfactory method in scenarios of limited availability of more accurate tests. However, further studies are needed to assess the sensitivity/specificity of the method, as well as standardization in the collection and interpretation of the findings.

\section{EPV111/\#303 SENTINEL LYMPH NODE MAPPING FOR ENDOMETRIAL CANCER: A PROSPECTIVE STUDY ABOUT THIRTY EIGHT CASES}

${ }^{1} S$ Sghaier*, ${ }^{2} \mathrm{H}$ Bouaziz, ${ }^{3} \mathrm{~N}$ Tounsi, ${ }^{2} \mathrm{M}$ Bouhani, ${ }^{4} \mathrm{~L}$ Zaabar, ${ }^{5} \mathrm{G}$ Sahraoui, ${ }^{2} \mathrm{H}$ Bouzainne, ${ }^{2} \mathrm{M}$ Slimane, ${ }^{2} \mathrm{~K}$ Rahal. ${ }^{1}$ Salah Azaiez Institute, Surgical Oncology Department, Tunis, Tunisia; ${ }^{2}$ Salah Azaiez Institute of Oncology, Surgical Oncology, Tunis, Tunisia; ${ }^{3}$ salah Azaiz Institut, Department of Surgical Oncologists, Tunis, Tunisia; ${ }^{4}$ Salah Azaiez Institute, Nuclear Medicine, Tunis, Tunisia; ${ }^{5}$ Salah Azaiez Institute, Anatomopathology, Tunis, Tunisia

\subsection{6/ijgc-2021-IGCS.181}

Objectives Endometrial cancer (EC) represents the most common gynecological cancer mostly diagnosed at stage I. Sentinel lymph node (SLN) arised as a valuable option to lymph node dissection. We aim to determine negative predictive value (NPV), overall and bilateral detection rates of SLN in EC stage I.
Methods This was a cross-sectional prospective study including 38 patients with EC stage I treated at Salah Azaiz Institute over a period of 34 months from March 2018 to January 2021.

Results Endometrioid adenocarcinoma was reported in $89 \%$ of cases. The pelvic MRI showed IA and IB stages in 58\% and $42 \%$ of cases, respectively.The detection techniques were combined (48\%), colorimetric (34\%) and radioisotope (18\%). Lymphoscintigraphy was conducted in $66 \%$ of women demonstrating overall, bilateral and failed detection rates of $92 \%$, $24 \%$ and $8 \%$, respectively. The overall, bilateral and failed intra-operative detection rates were of $76 \%, 37 \%$ and $24 \%$ respectively. A micrometastasis (1\%) was noted among a total of 87 SLNs. False negative rate (FNR) and NPV were of $0 \%$ and $100 \%$.Factors affecting overall detection wereinitial histologic grade $(p=0.01)$ and tumor size on MRI $(p=0.04)$. Final histologic grade $1(p=0.005), 2(p=0.002)$ and myometrial invasion $(\mathrm{p}=0.04)$ were also significant contributors. No significant factors affecting bilateral detection were set.

Conclusions FNR and NPV were of $0 \%$ and $100 \%$ similarly to previous results through literature. We aim to continue this promising protocol toward including more patients that may helps us improve our overall and bilateral detection rates.

\section{EPV112/\#318 UTERINE CARCINOSARCOMA FOLLOWING TAMOXIFEN THERAPY FOR BREAST CANCER: A SERIES OF 11 CASES}

K Ben Hamida*, L Naija, M Ghalleb, MA Bouida, R Chargui, K Rahal. Salah Azaiez Institute of Oncology, Surgical Oncology Department, Tunis, Tunisia

\subsection{6/ijgc-2021-IGCS. 182}

Objectives Uterine carcinosarcoma (UC) is rare and carries a poor prognosis. It represents only $1-2 \%$ of uterine cancers and less than $5 \%$ of all uterine malignancies. Although these tumors usually arise de novo, some cases developed under Tamoxifen therapy have been reported. We aim to report our institution's experience.

Methods A retrospective study of 11 women with endometrial carcinosarcoma after breast cancer were treated at Salah Azaiez Institute of Oncology from 2004 to 2014.

Results The mean age of UC diagnosis was 64 years (50-82 years). All patients were given adjuvant hormone therapy by Tamoxifen for breast cancer. The mean duration of Tamoxifen use was 42 months (3-60 months) with a mean cumulative dose of $25709 \mathrm{mg}$ (1800-36500mg). The main presenting symptom of UC was post-menopausal bleeding. Ultrasound showed thickened endometrium in four cases. Endometrial biopsy revealed UC in three cases. Surgery was performed in ten cases. It consisted of total hysterectomy and bilateral oophorectomy in all cases; we performed lymphadenectomy in three cases. Adjuvant chemotherapy and radiotherapy were performed in three cases. The median follow-up after surgery was nine months (1-64 months). One patient developed a peritoneal recurrence five months after surgery. Three women developed bone metastasis from their uterine cancer, and two patients developed liver metastases from their breast cancer.

Conclusions The survival benefits associated with five-year adjuvant Tamoxifen counterbalances the low morbidity and mortality risk associated with endometrial adenocarcinoma development. Things are different with UC and its pejorative 
prognosis. The rarity of this tumor makes the risk of its development undetermined.

\section{EPV113/\#323 PROGNOSTIC FACTORS AND ONCOLOGIC OUTCOMES FOR PATIENTS TREATED WITH ADJUVANT CHEMOTHERAPY AND VAGINAL VAULT BRACHYTHERAPY FOR STAGE I ENDOMETRIAL SEROUS CARCINOMAS}

${ }^{1} \mathrm{~V}$ Dorion*, ${ }^{2} \mathrm{~V}$ Samouëlian, ${ }^{1} \mathrm{M}-\mathrm{C}$ Beauchemin, ${ }^{2} \mathrm{~B}$ Cormier, ${ }^{2} \mathrm{E}$ Tremblay, ${ }^{1} \mathrm{M}$ Barkati. ${ }^{1}$ Centre hospitalier de I'Université de Montréal, Radiation Oncology, Montreal, Canada; ${ }^{2}$ Gynecologic Oncology Service, CHUM, Université de Montréal, Department of Obstetrics and Gynecology, Montreal, Canada

\subsection{6/ijgc-2021-IGCS. 183}

Objectives Endometrial serous carcinomas (ESC) hold a poor prognosis, even at early stages. This study evaluates the outcomes and prognostic factors for stage I (FIGO 2018) ESC treated with adjuvant chemotherapy and vaginal vault brachytherapy (VBT).

Methods Patients were selected through a database of patients treated with hysterectomy for stage I ESC between 2007 and 2019 at the Centre Hospitalier de l'Université de Montréal. The intended adjuvant treatment had to be 6 cycles of Carboblatin and Paclitaxel and VBT. Time to events were analyzed by Kaplan-Meier. Cox regression analysis was performed to identify prognostic factors.

Results A total of 76 patients with stage IA $(\mathrm{N}=64)$ and IB $(\mathrm{N}=12)$ ESC were included in this study. Median age at diagnostic was 67. Median follow up was 60 months. 5-year overall survival (OS) and progression-free survival (PFS) were 83\% and $79.5 \%$. Nine patients relapsed, 3 with local recurrence, 3 with regional recurrence and the other 3 with distant recurrence. Amongst the known prognostic factors included in univariate analysis, positive peritoneal washing and advanced age were significant prognostic factors for OS $(p<0.0001$ and $\mathrm{p}=0.013$, respectively). Age, isthmus invasion, deep myometrial invasion and positive peritoneal washings were significant prognostic factors for PFS $(p=0.049, p=0.024, p=0.022$ and $\mathrm{p}<0.0001$, respectively).

Conclusions In stage I ESC, adjuvant chemotherapy and VBT was associated with good oncologic outcomes. Advanced age and positive peritoneal washings were significant prognostic factors for OS. Further studies are needed to assess whether a subgroup of patients would benefit from treatment intensification or de-escalation.

\section{EPV114/\#325 EXPRESSION OF AQUAPORINS IN HUMAN ENDOMETRIAL CANCER: IDENTIFICATION AND REGULATION BY OVARIAN HORMONES IN CARCINOGENESIS OF ENDOMETRIAL CANCER}

${ }^{1} S$ Khan*, ${ }^{1} \mathrm{~A}$ Yool, ${ }^{2} \mathrm{C}$ Ricciardelli. 'Adelaide Medical School, Univeristy of Adelaide, Physiology, Australia/Adelaide, Australia; ${ }^{2}$ Adelaide Medical School, Univeristy of Adelaide, Discipline of Obstetrics and Gynaecology, Robinson Research Institute, Australia/Adelaide, Australia

\subsection{6/ijgc-2021-IGCS. 184}

Objectives Aaquaporins contribute to pathogenesis of Endometrial cancer. Our study presents the first screen of grade I and grade III endometrial cancer cell lines for all 13 AQP classes in response to physiological doses of estrogen and progesterone.

Methods Ishikawa (IKC, grade I) and MFE-280 (grade III) were assessed with estrogen and progesterone at relevant doses, at multiple time points for cell proliferation, motility (3D migration and invasion assays), and cytoskeletal organisation. Patterns of AQP expression were compared in IKC and MFE-280 by quantitative (q) PCR and western blot (WB).

Results Cell numbers, 3D migration and invasiveness were increased in IKC by estrogen and decreased by progesterone in a dose- and time-dependent manner. Estrogen induced formation of lamellipodia in IKC. The EC50 and IC50 values for estrogen and progesterone were $1 \mathrm{nM}$ and $100 \mathrm{nM}$ respectively. Transcript levels of AQPs $0,-2,-3,-4,-5,-8$ were significantly decreased by estrogen and progesterone in IKC, whereas AQP11 and AQP12 were increased. In contrast, in MFE-280 cells, estrogen and progesterone caused an increase in transcript levels for AQPs 3,-4,-7, -8, whereas expression of AQPs 0 , and -11 were decreased. Protein expression of AQP-1 and -4 was confirmed by WB.

Conclusions These findings indicate the potential role of aquaporins in progression and invasion of endometrial cancer, and highlight the previously unstudied AQPs 11 and 12 as targets of potential interest. Outcomes here provide a foundation for further exploration of aquaporin inhibitors in decreasing the progression of EC, and insights into new therapeutic strategies

\section{EPV115/\#333 INTERIM ANALYSIS OF 10-YEAR DATA REGARDING PRESENTATION AND MANAGEMENT OF UTERINE CARCINOSARCOMA (UCS) CASES ACROSS THE THAMES VALLEY CANCER ALLIANCE NETWORK}

${ }^{1}$ A Gkorila* ${ }^{2} S$ Smyth ${ }^{3} \mathrm{P}$ Tupper ${ }^{4} \mathrm{~A}$ Mcculloch ${ }^{1} \mathrm{~N}$ Sadeghi, ${ }^{1} \mathrm{~V}$ Le Thanh, ${ }^{1} \mathrm{~A}$ Sattar ${ }^{1} \mathrm{~S}$ Spencer, ${ }^{1} \mathrm{~K}$ Zarrindej, ${ }^{2} \mathrm{M}$ Abdalla, ${ }^{2} \mathrm{~S}$ Addley, ${ }^{1} \mathrm{~J}$ Rencher, ${ }^{1} \mathrm{G}$ Sharma, ${ }^{1} \mathrm{~A}$ Kashif, ${ }^{5} \mathrm{~S}$ Siddiki, ${ }^{2} \mathrm{M}$ Alazzam, ${ }^{2} \mathrm{H}$ Soleymani Majd. ${ }^{1} \mathrm{Oxford}$ University Hospitals NHS Foundation Trust, Obstetrics and Gynaecology, Oxford, UK; ${ }^{2}$ Oxford University Hospitals NHS Foundation Trust, Gynaecological Oncology, Oxford, UK; ${ }^{3}$ Buckinghamshire Healthcare NHS Trust, Obstetrics and Gynaecology, Aylesbury, UK; ${ }^{4}$ Royal Berkshire Hospital NHS Foundation Trust, Obstetrics and Gynaecology, Reading, UK; ${ }^{5}$ Great Western Hospitals NHS Foundation Trust, Obstetrics and Gynaecology, Swindon, UK

\subsection{6/ijgc-2021-IGCS. 185}

Objectives UCS comprises $<5 \%$ of uterine malignancies, accounting for $>15 \%$ associated mortality. With no established guidelines, we present our experience to determine clinical characteristics, treatment modalities and histology outcomes of UCS.

Methods We conducted a multicentre retrospective cohort study, including all surgically managed UCS cases between March 2010 and January 2020. Data was collected on patients' demographics, medical history, pre-operative and final histology and FIGO staging, peri-operative and post-operative findings.

Results 82 (9.7\%) UCS cases were identified from a total of 847 surgically managed uterine cancers, with 51 diagnosed with UCS. 3 cases were down and 12 up-staged following surgery. 15 cases of MRI lymphadenopathy led to a PPV of $40 \%$. Positive lymph nodes and omentum were identified in $15.8 \%$ and $11.3 \%$ of cases respectively, with half of lymph node metastases diagnosed following systematic dissection (the majority of which were LVSI positive). There were no operative complication themes. 